July 1995

\title{
The Lyapunov Spectrum of a Continuous Product of Random Matrices
}

\author{
Andrea Gambat \\ Dipartimento di Matematica, Politecnico di Torino, 10129 Turin, Italy \\ and INFN, sezione di Milano, 20133 Milan, Italy \\ Igor V. Kolokolov P \\ Budker Institute of Nuclear Physics, 630090 Novosibirsk, Russia.
}

\begin{abstract}
We expose a functional integration method for the averaging of continuous products $\hat{P}_{t}$ of $N \times N$ random matrices. As an application, we compute exactly the statistics of the Lyapunov spectrum of $\hat{P}_{t}$. This problem is relevant to the study of the statistical properties of various disordered physical systems, and specifically to the computation of the multipoint correlators of a passive scalar advected by a random velocity field. Apart from these applications, our method provides a general setting for computing statistical properties of linear evolutionary systems subjected to a white noise force field.
\end{abstract}

Key words: Lyapunov exponents, random matrices, functional integral, disordered systems, passive scalar, Gauss decomposition, loop groups.

\footnotetext{
${ }^{1}$ E-mail: gamba@pol88a.polito.it

${ }^{2}$ E-mail: kolokolov@vxinpb.inp.nsk.su
} 


\section{Introduction}

In this work we give a detailed exposition of a functional integral method for the averaging of time-ordered exponentials of $N \times N$ random matrices which has found several applications in the study of the statistical properties of disordered systems.

The method was introduced by one of the authors [2] in the $N=2$ case in order to compute the partition function of the Heisenberg ferromagnet, and was thereafter applied to the study of one-dimensional Anderson localization [3] and to some problems of mesoscopic physics [4]. Later, the same technique [6, 7] allowed to obtain analytical results in the problem of a passive scalar advected by a 2 -dimensional random velocity field. The approach of refs. [6, 7] was then extended to the more general $N$-dimensional case in ref. [1].

In the present work we expose the method in its full generality and show that it allows to compute exactly the statistics of the whole Lyapunov spectrum of the matrix $\hat{P}_{t}$ describing the time evolution of a linear system subjected to a white noise force field. Such a spectrum is relevant [8] in the computation of the multipoint correlators of the passive scalar (as well as in the computation of the correlators of passive vectors and tensors); in this case the matrix $\hat{P}_{t}$ describes the time evolution of particles in a turbulent fluid, linearized around a given trajectory.

However, our setting presents a high degree of generality and therefore a wider range of applications. In particular, our formalism can be naturally applied to the study of $N$ level quantum-mechanical systems affected by a random noise, and it is complementary to the supersymmetric approach [14, 15] to the problem of $N / 2$ channels localization in a disordered conductor.

\section{Averages of time-ordered exponentials}

Let us start with the problem of computing gaussian averages of the form

$$
\left\langle\mathcal{F}\left[\hat{P}_{t}\right]\right\rangle=\frac{1}{\mathcal{N}} \int \mathcal{D} \hat{X} \exp (-S[\hat{X}]) \mathcal{F}\left[\hat{P}_{t}\right]
$$

where $\hat{X}(s)$, for $0 \leq s \leq T$, is a traceless $N \times N$ hermitian matrix,

$$
\mathcal{D} \hat{X} \equiv \prod_{0 \leq s \leq T} \prod_{i<j} d X_{i j}(s) d X_{j i}(s) \prod_{i} d X_{i i}(s)
$$

is the Feynman-Kac measure, $\mathcal{N}$ is chosen in such a way that $\langle 1\rangle=1$,

$$
S[\hat{X}]=\frac{1}{4 D} \int_{0}^{T} \operatorname{Tr} \hat{X}^{2}(s) d s
$$

and $\hat{P}_{t}$ is the time-ordered exponential

$$
\hat{P}_{t}=\mathcal{T} \exp \left(\int_{0}^{t} \hat{X}(s) d s\right)
$$


such that $\mathbf{r}(t)=\hat{P}_{t} \mathbf{r}_{0}$ is the general solution of the linear problem $\dot{\mathbf{r}}=\hat{X} \mathbf{r}, \mathbf{r}(0)=\mathbf{r}_{0}$ and

$$
\dot{\hat{P}}_{t} \hat{P}_{t}^{-1}=\hat{X}
$$

We shall now introduce a set of "collective" integration variables in order to re-express (『) in a more tractable form. At the same time, we shall chose the new variables in such a way that (3) still be quadratic and that the Jacobian determinant of the functional transformation be particularly simple.

As a first step, let us Gauss-decompose the matrix $\hat{P}_{t}$ :

$$
\hat{P}_{t}=(\mathbf{1}+\hat{\phi}(t)) \cdot \exp (\hat{\tau}(t)) \cdot(\mathbf{1}+\hat{\theta}(t))
$$

where

$$
\begin{aligned}
\phi_{i j}(t) & \equiv 0, \quad i \leq j \\
\theta_{i j}(t) & \equiv 0, \quad i \geq j \\
\tau_{i j}(t) & \equiv \tau_{i}(t) \delta_{i j} \\
\tau_{N}(t) & \equiv-\sum_{j=1}^{N-1} \tau_{j}(t)
\end{aligned}
$$

Moreover, in order to ensure the equality $\hat{P}_{0}=\mathbf{1}$ we shall impose

$$
\hat{\phi}(0)=\mathbf{0}, \quad \hat{\theta}(0)=\mathbf{0}
$$

We would like now to re-express the "local" degrees of freedom $X_{i j}(t)$ in terms of the global ones $\phi_{i j}(t), \tau_{i j}(t), \theta_{i j}(t)$. This can be accomplished by making use of the basis $\hat{e}_{i j}$ of the matrix algebra, which is defined by $\left(\hat{e}_{i j}\right)_{k l}=\delta_{i k} \delta_{j l}$ and satisfies the commutation rules

$$
\left[\hat{e}_{i j}, \hat{e}_{k l}\right]=\delta_{j k} \hat{e}_{i l}-\delta_{i l} \hat{e}_{k j}
$$

In particular, one has

$$
\begin{aligned}
& \hat{e}_{i i} \hat{e}_{k l}=\hat{e}_{k l}\left(\hat{e}_{i i}+\delta_{i k}-\delta_{i l}\right) \\
& e^{\hat{\tau}} \hat{e}_{i j} e^{-\hat{\tau}}=e^{\tau_{i}-\tau_{j}} \hat{e}_{i j}
\end{aligned}
$$

From these relations the desired expression for $X_{i j}$ readily follows:

$$
\begin{aligned}
X_{i j}= & \dot{\phi}_{i j}+\sum_{k} \dot{\phi}_{i k} \tilde{\phi}_{k j} \\
& +\dot{\tau}_{i} \delta_{i j}+\phi_{i j} \dot{\tau}_{j}+\dot{\tau}_{i} \tilde{\phi}_{i j}+\sum_{k} \phi_{i k} \dot{\tau}_{k} \tilde{\phi}_{k j} \\
& +A_{i j}+\sum_{k}\left(\phi_{i k} A_{k j}+A_{i k} \tilde{\phi}_{k j}\right)+\sum_{k, l} \phi_{i k} A_{k l} \tilde{\phi}_{l j}
\end{aligned}
$$

where $A_{i j} \equiv e^{\tau_{i}-\tau_{j}} \sum_{k} \dot{\theta}_{i k}\left(\delta_{k j}+\tilde{\theta}_{k j}\right)$,

$$
\tilde{\phi}_{i j} \equiv-\phi_{i j}+\sum_{k} \phi_{i k} \phi_{k j}-\sum_{k, l} \phi_{i k} \phi_{k l} \phi_{l j}+\cdots
$$


and a similar definition holds for $\tilde{\theta}_{i j}$ (for any fixed $N$ (13) is a finite sum, since $\hat{\phi}$ is a nilpotent matrix; the same is true for $\hat{\theta}$ ).

Substituting (12) in (3) one obtains

$$
\frac{1}{2} \operatorname{Tr} \hat{X}^{2}=\frac{1}{2} \sum_{j=1}^{N} \dot{\tau}_{j}^{2}+\sum_{i, j}\left(\dot{\phi}_{i j}+\sum_{k=1}^{N} \tilde{\phi}_{i k} \dot{\phi}_{k j}\right) e^{\tau_{j}-\tau_{i}}\left(\sum_{k=1}^{N} \dot{\theta}_{j l} \tilde{\theta}_{l i}+\dot{\theta}_{j i}\right)
$$

The form of (14) suggests the introduction of the new variables

$$
\begin{aligned}
& \bar{\phi}_{i j}=\sum_{k, l} \dot{\theta}_{i k}\left(\delta_{k l}+\tilde{\theta}_{k l}\right)\left(\delta_{l j}+\tilde{\phi}_{l j} e^{\tau_{j}-\tau_{l}}\right) e^{\tau_{i}-\tau_{j}}, \quad i<j \\
& \bar{\phi}_{i j} \equiv 0, \quad i \geq j
\end{aligned}
$$

so that

$$
\frac{1}{2} \operatorname{Tr} \hat{X}^{2}=\frac{1}{2} \sum_{j=1}^{N} \dot{\tau}_{j}^{2}+\sum_{i, j} \dot{\phi}_{i j} \bar{\phi}_{j i}
$$

Relation (15) can be inverted giving

$$
\dot{\theta}_{i j}=\sum_{k, l} \bar{\phi}_{i k} e^{\tau_{k}-\tau_{i}}\left(\delta_{k l}+\phi_{k l} e^{\tau_{l}-\tau_{k}}\right)\left(\delta_{l j}+\theta_{l j}\right) \chi_{l i}
$$

where

$$
\chi_{i j} \equiv 1-\bar{\chi}_{i j} \equiv \begin{cases}1, & i>j \\ 0, & i \leq j\end{cases}
$$

Through (17) one can re-express the $\theta_{i j}$ as functions of the new variables $\phi_{i j}, \tau_{i}$ and $\bar{\phi}_{i j}$ in a recursive way, thanks to the "triangular" form of the equation. For instance, for $N=3$ one gets

$$
\begin{aligned}
\theta_{23}(t) & =\int_{0}^{t} \bar{\phi}_{23}(s) e^{-\tau_{1}(s)-2 \tau_{2}(s)} d s \\
\theta_{12}(t) & =\int_{0}^{t} A(s) d s, \quad \text { where } \quad A=\left(\bar{\phi}_{12}+\bar{\phi}_{13} \phi_{32}\right) e^{\tau_{2}-\tau_{1}} \\
\theta_{13}(t) & =\int_{0}^{t}\left[A(s) \theta_{23}(s)+\bar{\phi}_{13}(s) e^{-2 \tau_{1}(s)-\tau_{2}(s)}\right] d s
\end{aligned}
$$

As a matter of fact, for any fixed $i$ the $N-i$ functions $\theta_{i j}$ can be expressed through the $N-i-1$ functions $\theta_{i+1, j}$ and the remaining variables by means of a single quadrature. This is an important point, since for practical calculations $\hat{P}_{t}$ has to be re-expressed in terms of the new variables $\hat{\phi}, \dot{\hat{\tau}}, \hat{\bar{\phi}}$.

We must now substitute $\hat{X}(s)$ as an integration variable in the functional integral (1) with the new variables $\hat{\phi}(s), \dot{\hat{\tau}}(s), \hat{\bar{\phi}}(s)$. Again using the commutation rules (10), and renaming $\dot{\tau}_{i} \equiv \rho_{i}$ for convenience, we finally get

$$
\begin{aligned}
X_{i j} & =\bar{\phi}_{i j}+\sum_{k} \phi_{i k} \bar{\phi}_{k j}, \quad i<j \\
X_{i i} & =\rho_{i}+\sum_{k}\left(\phi_{i k} \bar{\phi}_{k i}-\bar{\phi}_{i k} \phi_{k i}\right), \quad i=1, \ldots, N
\end{aligned}
$$




$$
\begin{aligned}
X_{i j} & =\phi_{i j} \rho_{j}+\rho_{i} \tilde{\phi}_{i j}+\dot{\phi}_{i j} \\
& +\sum_{k}\left(\phi_{i k} \rho_{k} \tilde{\phi}_{k j}+\dot{\phi}_{i k} \tilde{\phi}_{k j}+\phi_{i k} \bar{\phi}_{k j}-\bar{\phi}_{i k} \phi_{k j}\right) \\
& -\sum_{k, l}\left(\bar{\chi}_{j k} \phi_{i k} \bar{\phi}_{k l} \phi_{l j}+\bar{\chi}_{l i} \bar{\phi}_{i k} \phi_{k l} \tilde{\phi}_{l j}\right) \\
& -\sum_{k, l, m} \bar{\chi}_{m k} \phi_{i k} \bar{\phi}_{k l} \phi_{l m} \tilde{\phi}_{m j}, \quad i>j
\end{aligned}
$$

In ref. [1] the $N=3$ case was explicitly considered. We observe that the matrix elements of $\hat{X}(t)=\hat{P}_{t} \hat{P}_{t}^{-1}$ transform as

$$
X_{i j}(t) \rightarrow e^{\sigma_{i j}} X_{i j}(t)
$$

under the global gauge transformation

$$
\phi_{i j}(t) \rightarrow e^{\sigma_{i j}} \phi_{i j}(t), \quad \bar{\phi}_{i j}(t) \rightarrow e^{\sigma_{i j}} \bar{\phi}_{i j}(t), \quad \rho_{i}(t) \rightarrow \rho_{i}(t)
$$

where $\sigma_{i j}$ satisfies $\sigma_{i k}+\sigma_{k j}=\sigma_{i j}, \sigma_{i j}=-\sigma_{j i}$.

As the last step, we must compute the Jacobian determinant of the functional transformation (21). Notice first that through the shift given by

$$
\begin{aligned}
\bar{\phi}_{i j} & \rightarrow \bar{\phi}_{i j}-\sum_{k=1}^{N} \phi_{i k} \bar{\phi}_{k j} \\
\rho_{i} & \rightarrow \rho_{i}-\sum_{k=1}^{N}\left(\phi_{i k} \bar{\phi}_{k i}-\bar{\phi}_{i k} \phi_{k i}\right)
\end{aligned}
$$

(which has Jacobian $\mathcal{J}^{\prime}=1$ ) one can reduce to the computation of

$$
\operatorname{Det}\left(\frac{\delta \hat{X}_{-}}{\delta \hat{\phi} \delta \hat{\rho} \delta \hat{\bar{\phi}}}\right)
$$

where $\hat{X}_{-}$is the strictly lower triangular part of $\hat{X}$. The Jacobian (25) can be computed by means of the standard regularization procedure (see ref. [2])

$$
\begin{aligned}
& \hat{\phi}_{n}=\hat{\phi}\left(t_{n}\right), \quad \hat{\rho}_{n}=\hat{\rho}\left(t_{n}\right), \quad \hat{\bar{\phi}}_{n}=\hat{\bar{\phi}}\left(t_{n}\right) \\
& t_{n}=h n, \quad n=1, \ldots, M, \quad h=T / M \rightarrow 0, \quad M \rightarrow+\infty \\
& \dot{\hat{\phi}}(t) \rightarrow \frac{\hat{\phi}_{n}-\hat{\phi}_{n-1}}{h}, \quad \hat{\phi}(t) \rightarrow \frac{\hat{\phi}_{n}+\hat{\phi}_{n-1}}{2}
\end{aligned}
$$

giving

$$
\mathcal{J} \propto \exp \left(\sum_{j=1}^{N-1}(N-j) \int_{0}^{T} \rho_{j}(s) d s\right)
$$

Applying now the variable transformation $\hat{X} \rightarrow(\hat{\phi}, \hat{\rho}, \hat{\bar{\phi}})$ one sees that the functional integral (1) reduces to

$$
\left\langle\mathcal{F}\left[\hat{P}_{t}\right]\right\rangle=\frac{1}{\mathcal{N}^{\prime}} \int \mathcal{D} \hat{\phi} \mathcal{D} \hat{\rho} \mathcal{D} \hat{\bar{\phi}} \exp \left(-S^{\prime}[\hat{\phi}, \hat{\rho}, \hat{\bar{\phi}}]\right) \mathcal{F}\left[(\mathbf{1}+\hat{\phi}) e^{\hat{\tau}}(\mathbf{1}+\hat{\theta})\right]
$$


where $\hat{\theta}=\hat{\theta}[\hat{\phi}, \hat{\rho}, \hat{\bar{\phi}}]$ is obtained by solving (17), $\hat{\tau}=\int_{0}^{T} \hat{\rho}(s) d s$, the $\rho_{i}$ are constrained by (8), $\mathcal{N}^{\prime}$ is the normalization factor and

$$
S^{\prime}=\frac{1}{2 D} \int_{0}^{T}\left(\frac{1}{2} \sum_{k=1}^{N} \rho_{k}^{2}+\sum_{i, j} \dot{\phi}_{i j} \bar{\phi}_{j i}-2 D \sum_{k=1}^{N-1}(N-k) \rho_{k}\right) d s
$$

In (11) the functional integration is constrained to the surface

$$
\Gamma_{0}=\left\{X_{i j}(s)=X_{j i}^{*}(s), \quad 0 \leq s \leq T\right\}
$$

In refs. [2, 7] it was shown, using the Cauchy theorem, that whenever $\mathcal{F}$ is holomorphic in the matrix elements $X_{i j}$ one can modify the integration surface $\Gamma_{0}$ to the homotopically equivalent

$$
\Gamma_{1}=\left\{\phi_{i j}(s)=\bar{\phi}_{j i}^{*}(s), \operatorname{Im} \rho_{i}(s)=0, \quad 0 \leq s \leq T\right\}
$$

without affecting the value of the integral. This means that in (28) $\hat{\bar{\phi}}$ may be regarded as the hermitian conjugate of $\hat{\phi}$.

We would like to remark that expressions similar to (21) were obtained in the framework of conformal field theory [16]. However, the explicit form of the variables $\bar{\phi}$ and of the Jacobian $\mathcal{J}$, which are essential for any physical application of our method, were not computed there.

\section{The Lyapunov Spectrum}

We shall now define the Lyapunov exponents $\lambda_{j}, j=1, \ldots, N$, according to the relation [9]

$$
\lambda_{1}+\cdots+\lambda_{k}=\frac{1}{T} \log \operatorname{Vol}\left(\hat{P}_{T} \mathbf{v}_{1}, \ldots, \hat{P}_{T} \mathbf{v}_{k}\right)
$$

where $\mathbf{v}_{1}, \ldots, \mathbf{v}_{k}$ is an orthonormal set of vectors generating a unitary $k$-volume. For the sake of definiteness we shall choose $\mathbf{v}_{j}=\mathbf{e}_{j}$, where $\left(\mathbf{e}_{j}\right)_{i}=\delta_{i j}$. One has

$$
\operatorname{Vol}\left(\hat{P}_{T} \mathbf{e}_{1}, \ldots, \hat{P}_{T} \mathbf{e}_{k}\right)=\sqrt{\sum_{a=1}^{l_{k}} \Delta_{a}^{2}\left(\hat{M}_{k}\right)}
$$

where $\Delta_{a}\left(\hat{M}_{k}\right), a=1, \ldots, l_{k}, l_{k} \equiv\left(\begin{array}{l}n \\ k\end{array}\right)$ are the $k \times k$ minors of the $n \times k$ matrix $\hat{M}_{k}=$ $\left[\hat{P}_{T} \mathbf{e}_{1}, \ldots, \hat{P}_{T} \mathbf{e}_{k}\right]$. Let $\vec{\phi}_{j}, \mathbf{p}_{j}, j=1, \ldots, N$ be the vectors defined by $\left(\vec{\phi}_{j}\right)_{i}=\delta_{i j}+\phi_{i j}$, $\left(\mathbf{p}_{j}\right)_{i}=\left(\hat{P}_{T}\right)_{i j}$. Then

$$
\begin{aligned}
\mathbf{p}_{j} & =\sum_{i, k}\left(\delta_{i k}+\phi_{i k}\right) e^{\tau_{k}}\left(\delta_{k j}+\theta_{k j}\right) \mathbf{e}_{i} \\
& =\sum_{k=1}^{N} e^{\tau_{k}}\left(\delta_{k j}+\theta_{k j}\right) \vec{\phi}_{k} \\
& =e_{j}^{\tau} \vec{\phi}_{j}+\sum_{k<j} \theta_{k j} e^{\tau_{k}} \vec{\phi}_{k}
\end{aligned}
$$


From (34) and the multilinearity of determinants it follows

$$
\begin{aligned}
\Delta_{a}\left(M_{k}\right) & =\Delta_{a}\left[\mathbf{p}_{1}, \ldots, \mathbf{p}_{n}\right] \\
& =\Delta_{a}\left[e^{\tau_{1}} \vec{\phi}_{1}, \ldots, e^{\tau_{k}} \vec{\phi}_{k}\right] \\
& =e^{\tau_{1}+\cdots+\tau_{l}}\left(\delta_{a, 1}+\sum_{r_{i j} \geq 1} c_{i j}^{a, k} \phi_{i j}^{r_{i j}}\right)
\end{aligned}
$$

where $\Delta_{1}$ is the minor obtained from the first $k$ rows of $M_{k}, r_{i j}$ are strictly positive integers, and $c_{i j}^{a, k}$ are integer coefficients with $c_{i j}^{1, k} \equiv 0$. One has then

$$
\begin{aligned}
\lambda_{1}+\cdots+\lambda_{k} & \left.=\frac{1}{T}\left[\tau_{1}(T)+\cdots+\tau_{k}(T)\right)\right] \\
& +\frac{1}{2 T} \log \left[1+\sum_{a=2}^{l_{k}}\left(\sum_{r_{i j} \geq 1} c_{i j}^{a, k} \phi_{i j}^{r_{i j}}(T)\right)^{2}\right]
\end{aligned}
$$

and

$$
\lambda_{k}=\frac{1}{T} \int_{0}^{T} \rho_{k} d t+\frac{1}{2 T}\left[\log \left(1+f_{k}(\hat{\phi})\right)-\log \left(1+f_{k-1}(\hat{\phi})\right)\right]
$$

where $1+f_{k}(\hat{\phi})$ is the argument of the logarithm in (36).

Let us now compute the probability distribution function for $\lambda_{k}$. The form of (29) implies that the $\phi$-dependent terms in (37) give no contribution, since they do not contain the conjugate variables $\bar{\phi}_{i j}$.

We are therefore left with $N-1$ Gaussian integrations over $\rho_{1}, \ldots, \rho_{k-1}, \rho_{k+1}, \ldots, \rho_{N}$ which give the following exact result for the statistics of $\rho_{k}$ :

$$
\mathcal{D} \rho_{k} \exp \left(-\frac{N}{4 D(N-1)} \int_{0}^{T}\left(\rho_{k}(s)-\bar{\lambda}_{k}\right)^{2} d s\right)
$$

where

$$
\bar{\lambda}_{k}=D(N-2 k+1), \quad k=1, \ldots, N
$$

The probability distribution function $p\left(\lambda_{k} ; T\right)$ of the $k$-th Lyapunov exponent $\lambda_{k}$ is then:

$$
p\left(\lambda_{k} ; T\right)=\frac{1}{2} \sqrt{\frac{N T}{\pi D(N-1)}} \exp \left(-\frac{N T}{4 D(N-1)}\left(\lambda_{k}-\bar{\lambda}_{k}\right)^{2}\right)
$$

The Lyapunov exponents $\lambda_{k}$ are statistically dependent due to the constraint (8) and their joint distribution function has a generalized Gaussian form [1. We have thus obtained a complete knowledge of the statistics of the Lyapunov spectrum of the matrix $\hat{P}_{t}$. This has an essential application to the problem of the computation of the multipoint correlators of a passive scalar advected by a random velocity field (see the Appendix).

\footnotetext{
${ }^{3}$ The Gaussian distribution of the Lyapunov exponent in the $N=2$ case was obtained in the context of the passive scalar problem in ref. [6].
} 


\section{Conclusions}

In this work we gave a detailed exposition in the general $N \times N$ case of a functional integral method for the averaging of time-ordered exponentials of random matrices which has found several applications to the study of the statistical properties of disordered physical systems [2, 3, 4, 1, 6, 7, 8] and we have shown how the statistics of the Lyapunov spectrum of a linear evolutionary process can be computed exactly. As a matter of fact, our method provides a general setting for computing the statistics of linear evolutionary systems subjected to a white noise force field.

We would like to conclude with some remarks. The definition of the Lyapunov exponents as the logarithmic rate of growth of a $k$-dimensional parallelepiped (see eq. (32) and ref. [9]) is the most natural from a physical point of view, e.g. in the passive scalar problem. Generally speaking these exponents do not coincide with the logarithms of the eigenvalues of the

evolution matrix $\hat{P}_{t}$. The statistics of the eigenvalues of a similar evolution matrix was studied in refs. 110, 11, 12, 13]. Our method, however, allows one to obtain a more detailed statistical information about the evolution of initial vectors and to compute non-trivial correlation functions of their components. For an application to the passive scalar problem see ref. [8].

Lastly, we would like to remark that a more refined application of the functional integral method we described allowed to solve effectively the more difficult case of a "coloured" noise (see ref. [7]).

\section{Acknowledgments}

We gratefully acknowledge inspiring discussions with P. Casati, A. Cattaneo, M. Chertkov, B. Chirikov, G. Jona-Lasinio, F. Magri, M. Martellini, O. Ragnisco, P. Santini and A. Vulpiani. One of us (A.G.) would like to thank the Physics Department of Rome University $L a$ Sapienza, where part of this work was done, for warm hospitality.

\section{Appendix}

The method we exposed in this work has a direct application to the computation of the statistics of a scalar passively advected by a random velocity field. In order to illustrate this point we will briefly recall here the terms of the problem. For more detail see refs. [5, 6, 7, 11, 81.

The evolution of a scalar field $\theta(\mathbf{r}, t)$ passively advected by a velocity field $\mathbf{v}(\mathbf{r}, t)$ and generated by a source $\phi(\mathbf{r}, t)$ is given by

$$
\dot{\theta}+\mathbf{v} \cdot \nabla \theta=\phi
$$


If we impose on (41) the asymptotic condition $\theta(\mathbf{r},-\infty)=0$ we get the solution

$$
\theta(\mathbf{r}, t)=\int_{0}^{+\infty} \phi(\mathbf{R}(\mathbf{r}, t-s), t-s) d s
$$

saying that $\theta(\mathbf{r}, t)$ is completely determined in terms of the trajectories $\mathbf{R}\left(\mathbf{r}_{0}, t\right)$ of the fluid particles:

$$
\dot{\mathbf{R}}=\mathbf{v}(\mathbf{R}, t), \quad \mathbf{R}\left(\mathbf{r}_{0}, 0\right)=\mathbf{r}_{0}
$$

Let us now take $\phi$ and $\mathbf{v}$ to be random, $\delta$-correlated in time fields. The source $\phi$ will be assumed to be spatially correlated on a scale $L$. The velocity field $\mathbf{v}$ might be multi-scale, with smallest scale larger or of the order of $L$. The statistics of $\phi$ and $\mathbf{v}$ will be assumed to be spatially isotropic.

Generally speaking, one is interested in computing equal-time correlators of the form $\left\langle\theta\left(\mathbf{r}_{1}, 0\right) \theta\left(\mathbf{r}_{2}, 0\right)\right\rangle$ for $\left|\mathbf{r}_{2}-\mathbf{r}_{1}\right| \ll L$. From the isotropicity of the statistics of $\phi$ and $\mathbf{v}$ it follows that such quantities are rotation-invariant. Moreover, (42) implies that the statistics of $\theta$ is completely determined in terms of the statistics of the trajectories (43).

In order to subtract the effect or sweeping, let us chose a reference frame locally comoving with one of the fluid particles (see refs. [5, 6, 7). We can then locally linearize (43), obtaining

$$
\dot{\mathbf{R}} \simeq \hat{\sigma}(t) \mathbf{R}
$$

where $\sigma_{i j} \equiv \partial v_{j} / \partial r_{i}$, the matrix of velocity derivatives, will be taken to be a random gaussian process. In the general case we have $\hat{\sigma}=\hat{R}+\hat{S}$, where $\hat{R}$ is the antisymmetric part of $\hat{\sigma}$, inducing a rotation of the passive scalar blob, and $\hat{S}$ is the symmetric part, representing the stretching of the unit blob. We will consider the case of an incompressible fluid, so $\operatorname{Tr} \hat{S}=0$.

More specifically, let us consider the following statistics of $\hat{\sigma}$ :

$$
\begin{aligned}
& \mathcal{D} \mathcal{M}[\hat{\sigma}]=\mathcal{D} \hat{\sigma} \exp \left(-\frac{1}{2 D_{s}} \int_{0}^{T} \mathcal{L} d t\right) \\
& \mathcal{L}=\frac{1}{2}\left(\operatorname{Tr} \hat{S}^{2}-\frac{D_{s}}{D_{r}} \operatorname{Tr} \hat{R}^{2}\right)=\frac{1}{2} \operatorname{Tr}\left(\hat{S}+i \sqrt{\frac{D_{s}}{D_{r}}} \hat{R}\right)^{2}
\end{aligned}
$$

Since we are interested in rotation-invariant quantities the final result shall be independent on $D_{r}$. This arbitrariness allows one to substitute $\sqrt{D_{s} / D_{r}} \rightarrow-i, \hat{R} \rightarrow i \hat{R}, \hat{\sigma} \rightarrow \hat{X}=\hat{S}+i \hat{R}$ (see ref. [1]), and thus to consider a generic traceless hermitian matrix $\hat{X}$ with averaging weight $\exp \left(-\frac{1}{2 D_{s}} \int \frac{1}{2} \operatorname{Tr} \hat{X}^{2}\right)$ in the place of the generic traceless real matrix $\hat{\sigma}$ with the averaging weight (45): this allows one to refer to the results of sect. 2 .

From rotational invariance it follows that the statistics of $\theta$ is completely determined in terms of the statistics of the Lyapunov spectrum of the matrix $\hat{P}_{t}$ defined by

$$
\dot{\hat{P}}_{t}=\hat{X} \hat{P}_{t}, \quad \hat{P}_{0}=\mathbf{1}
$$

This reduces us to the problem studied in the preceding sections. The Gaussian statistics of the Lyapunov exponents agrees with an old result 17] about the Gaussian statistics of a line element in a $\delta$-correlated in time velocity field. 


\section{References}

[1] M. Chertkov, A. Gamba and I.V. Kolokolov, Phys. Lett. A 192 (94) 435

[2] I.V. Kolokolov, Phys. Lett. A 114 (86) 99; Ann. Phys. 202 (90) 165

[3] I.V. Kolokolov, JETP 76 (93) 1099; Europh. Letters 28 (94) 193

[4] A.S. Cattaneo, A. Gamba and I.V. Kolokolov, J. Stat. Phys. 76 (94) 1065

[5] G. Falkovich and V. Lebedev, Phys. Rev. E 49 (94) 1800

[6] M. Chertkov, Y.V. Fyodorov and I.V. Kolokolov, J. Phys. A 27 (94) 4925

[7] M. Chertkov, G. Falkovich, I. Kolokolov and V. Lebedev, Phys. Rev. E 51 (95) 5068

[8] E. Balkovskii, M. Chertkov, I. Kolokolov and V. Lebedev, JETP Letters 61 (95) 1049

[9] A. Crisanti, G. Paladin and A. Vulpiani, Products of Random Matrices in Statistical Physics, Springer-Verlag, Berlin-Heidelberg 1993, and references therein

[10] O.N. Dorokhov, JETP Lett. 36 (82) 318

[11] P.A. Mello, P. Pereyra and N. Kumar, Ann. Phys. 181 (88) 290

[12] C.W.J. Beenakker and B. Rejaei, Phys. Rev. Lett. 71 (93) 3689; Phys. Rev. B 49 (94) 7499

[13] M. Caselle, Phys. Rev. Lett. 74 (95) 2776

[14] K.B. Efetov, Adv. Phys. 32 (83) 53

[15] Y.V. Fyodorov and A.D. Mirlin, Phys. Rev. Lett. 67 (91) 2405

[16] A. Gerasimov, A. Marshakov, A. Morozov, M. Olshanetski and S. Shatashvili, Int. J. Mod. Phys. A 5 (90) 2495

[17] R.H. Kraichnan, J. Fluid Mech. 64 (74) 737 